\title{
The supraorbital eyebrow approach in children: clinical outcomes, cosmetic results, and complications
}

\author{
Brian J. Dlouhy, MD, ${ }^{1,2}$ Michael P. Chae, MBBS, ${ }^{2}$ and Charles Teo, MBBS, FRACS ${ }^{2}$ \\ 1Department of Neurosurgery, University of lowa Hospitals and Clinics, lowa City, lowa; and ${ }^{2}$ Centre for Minimally Invasive \\ Neurosurgery, Prince of Wales Hospital, Sydney, New South Wales, Australia
}

\begin{abstract}
OBJECT The supraorbital eyebrow approach utilizes an eyebrow skin incision to fashion a supraorbital craniotomy for exposure of the subfrontal corridor. This provides anterolateral access to surgical lesions in the anterior cranial fossa, parasellar regions, brainstem, and medial temporal lobe. With use of the endoscope, further areas can be accessed. This approach has been applied effectively in adults, but questions remain about its use in children-specifically with regard to adequate working space, effectiveness for achieving the desired results, cosmesis, and complications.

METHODS The authors conducted a retrospective review of more than 450 cases involving patients of all ages who had undergone a supraorbital eyebrow approach performed by the senior author (C.T.) from 1995 to 2013. Only cases involving patients younger than 18 years with a minimum follow-up of 6 weeks were included in this study. All inpatient and outpatient records were retrospectively reviewed and clinical/operative outcomes, cosmetic results, and complications were recorded. In the present article, the authors briefly describe the surgical approach and highlight any differences in applying it in children.
\end{abstract}

RESULTS Fifty-four pediatric patients who had undergone a supraorbital eyebrow approach met inclusion criteria. The pathological conditions consisted mostly of tumors or other resectable lesions. In a total of 51 resectable lesions, 44 surgeries resulted in a gross-total (100\%) resection and 7 cases resulted in subtotal (50\%-99\%) resection. The endoscope assisted and expanded visualization or provided access to areas not reached by standard microscopic visualization in all cases. Cosmetic outcomes were excellent. In all cases, the incisional scar was barely visible at 6 weeks. In 3 cases a minor bone defect was observed on the forehead. Given the small size of the frontal sinus in children, no frontal sinus breaches occurred. Additionally, no CSF leak or wound infection was identified.

CONCLUSIONS The supraorbital eyebrow approach is extremely effective in achieving desired results in properly selected cases in patients of all pediatric age ranges, from infants to teenagers. There is sufficient working space for the endoscope and all instruments, allowing for endoscopic assistance and bimanual surgical technique. Cosmetic results are excellent, and complications related to the approach are minimal.

http://thejns.org/doi/abs/10.3171/2014.10.PEDS1430

KEY WORDS supraorbital craniotomy; keyhole; minimally invasive surgery; eyebrow; neuroendoscopy; craniopharyngioma; complications; cosmesis; technique

$\mathrm{T}$ HE supraorbital eyebrow approach is a modification of a classic approach ${ }^{8}$ that utilizes an eyebrow skin incision and an anterolateral supraorbital craniotomy for exposure of the subfrontal corridor. ${ }^{27-29,32,33}$ This provides surgical access to the anterior cranial fossa floor, the basal frontal lobe, the frontal pole, the suprasellar and parasellar regions, the circle of Willis, the ventral brainstem, the proximal sylvian fissure, and the medial temporal lobe. The incision can be made either through the eyebrow ${ }^{32}$ or above it and can be described by the following terms: transciliary, ${ }^{34}$ transeyebrow, ${ }^{20}$ suprabrow, ${ }^{22}$ superciliary, $, 17,23,24$ and supraciliary. ${ }^{16}$ The orbital rim can be removed ${ }^{3,7,9,19,20,22,26,30,34}$ or preserved. . $^{6,14,16,21,27-29,33}$ In all, the approach relies on a small supraorbital craniotomy that is well positioned and relies on the keyhole concept, whereby a small cranial opening provides wider access to deeper structures. ${ }^{13,25}$

The supraorbital approach can be augmented by en-

ABBREVIATION PNET = primitive neuroectodermal tumor.

SUBMITTED January 20, 2014. ACCEPTED October 6, 2014.

INCLUDE WHEN CITING Published online November 14, 2014; DOI: 10.3171/2014.10.PEDS1430.

DISCLOSURE Dr. Teo reports a consultant relationship with Aesculap. 
doscopy, ${ }^{13,25}$ which enhances or expands visualization and access to the sella/pituitary fossa, region under the ipsilateral optic nerve/carotid artery, contralateral segments of the circle of Willis, anterior third ventricle, anterior interhemispheric fissure, upper third of the clivus, interpeduncular cistern, medial aspect of the ipsilateral middle cranial fossa and temporal lobe, and anterior olfactory groove. ${ }^{32,35}$

The supraorbital eyebrow approach has been described in the literature and has been used to treat unruptured aneurysms $s^{4,23,24,31,33}$ and various tumors ${ }^{10,14,35}$ in adults, such as meningiomas, pituitary adenomas, and intra-axial orbitofrontal masses, ${ }^{27}$ with good clinical outcomes. However, questions remain regarding its appropriateness in children due to several issues, which include feasibility of the approach, adequacy of the working space, effectiveness for achieving the desired results, cosmesis, and risk of complications. Therefore, we retrospectively examined all pediatric cases in which the senior author (C.T.) performed a supraorbital eyebrow craniotomy and evaluated clinical/ operative outcomes, cosmetic results, and complications.

\section{Methods}

\section{Patients}

A retrospective review identified more than 450 patients of all ages who had undergone a supraorbital eyebrow approach performed by the senior author (C.T.) from 1995 to 2013. Only patients younger than 18 years with at least 6 weeks' follow-up were included. A total of 54 patients met the age criterion and had immediate postoperative clinical follow-up, with postoperative imaging studies having been performed in 52 of these cases. Patients were diverse in ethnicity and came from the following countries: Australia, China, Peru, Cuba, Egypt, Vietnam, US, Indonesia, Germany, and Singapore. For all nonlocal patients, follow-up clinical examinations were conducted by neurosurgical colleagues who reported the results to the senior author; brain imaging studies were sent to the senior author. Otherwise, patients reported routinely to our clinic for follow-up, and imaging was performed at our hospital or the studies were brought in for review.

Postoperative cosmetic follow-up greater than 6 weeks was recorded in 42 of the 54 patients. Only physical examination by the senior author and postoperative photographs of the eyebrow and forehead of the patient were used in reporting the cosmetic outcome.

All inpatient and outpatient records were retrospectively reviewed, and the following clinical outcomes were recorded: patient age, sex, histopathological diagnosis, location of pathology, use of endoscope, and extent of tumor resection: gross-total resection $(100 \%)$, subtotal resection $(50 \%-99 \%)$, and partial resection $(<50 \%)$. The following cosmetic results were recorded: eyebrow alopecia, frontalis palsy, supraorbital hypoesthesia, and any forehead depression or indentation and titanium plate migration. The following complications were recorded: frontal sinus breach, CSF leak, wound infection, visual impairment, anosmia, diabetes insipidus, and any other major neurological complication or deficit.

\section{Surgical Technique}

The approach has been described in detail in the literature ${ }^{21,32,35}$ and is briefly described here, and any differences in children are highlighted (Fig. 1A-L). The child's head is placed in a 3-point rigid head-holder or placed on a padded headrest, gently translated anteriorly, extended, and rotated to the contralateral side. The ipsilateral eye is lubricated and closed with a temporary nylon tarsorrhaphy suture (Fig. 1C and D). Use of image-based frameless stereotactic guidance determines the position of the frontal sinus and ensures an optimal trajectory to the lesion of interest. It is prudent to avoid the frontal sinus. However, in children, the frontal sinus is often small and therefore generally not encountered. This is a positive aspect that makes the approach and craniotomy in children more standard and uniform than in adults.

A skin incision is made within the superior aspect of the eyebrow, extending from the supraorbital notch medially (to avoid the supraorbital nerve) to the lateral aspect of the eyebrow (Fig. 1C). We prefer to place the incision within the eyebrow, as it is more cosmetically pleasing when the scar is hidden within the eyebrow without loss of hair. The subgaleal layer is undermined, the skin is retracted superiorly with fish hooks, and a U-shaped pericranial flap is reflected inferiorly (Fig. 1E and F). Laterally, the superior portion of the temporalis muscle is dissected to allow placement of a bur hole below the superior temporal line in the keyhole region. A craniotomy is fashioned, and the protuberances of the orbital roof are drilled flat, as is the inner table of the inferior ledge of the craniotomy (Fig. 1E and F). The dura is then opened in a U-shaped fashion. The subfrontal corridor is dissected under microscopic visualization. With dissection of arachnoid adhesions and CSF egress, the frontal lobe falls away, an important aspect in the typically full brain of a child. Patience is required to prevent frontal lobe retraction and allow CSF egress. The pathology is identified and treated using the appropriate lesion-specific microsurgical techniques (Fig. $1 \mathrm{G}$ and $\mathrm{H}$ ). Neuroendoscopy with a $30^{\circ}$ endoscope may be used to augment visualization of the sella, interpeduncular cistern, interhemispheric cistern, contralateral circle of Willis, basal frontal lobe, and middle cranial fossa, if necessary (Fig. 2A-I). As in endonasal skull base surgery, where the long shaft of the endoscope is stabilized against sinonasal structures, in the supraorbital approach, the shaft can be stabilized by the superior or inferior bone edge of the craniotomy. In all cases, we use the endoscope to assist in visualization, and in operations undertaken for tumor resection, we use the endoscope to inspect for completeness of resection. This is often imperative in children. With the supraorbital approach, we never resect lesions through the endoscope's working channel. The endoscope is always used in an assistive manner.

After the lesion of interest has been addressed, the dura is closed in a watertight fashion and sutured to the lower rim of the craniotomy as an epidural tack-up (Fig. 1I). The bone is replaced with low-profile titanium plates (Fig. 1I). Care is taken to minimize the gap between the skull and bone flap at the more cosmetically noticeable superior margin. The pericranium, frontalis muscle, and galea are closed in layers. The skin of the eyebrow is reapproximated with a subcuticular nylon suture without knots (Fig. 1J). 

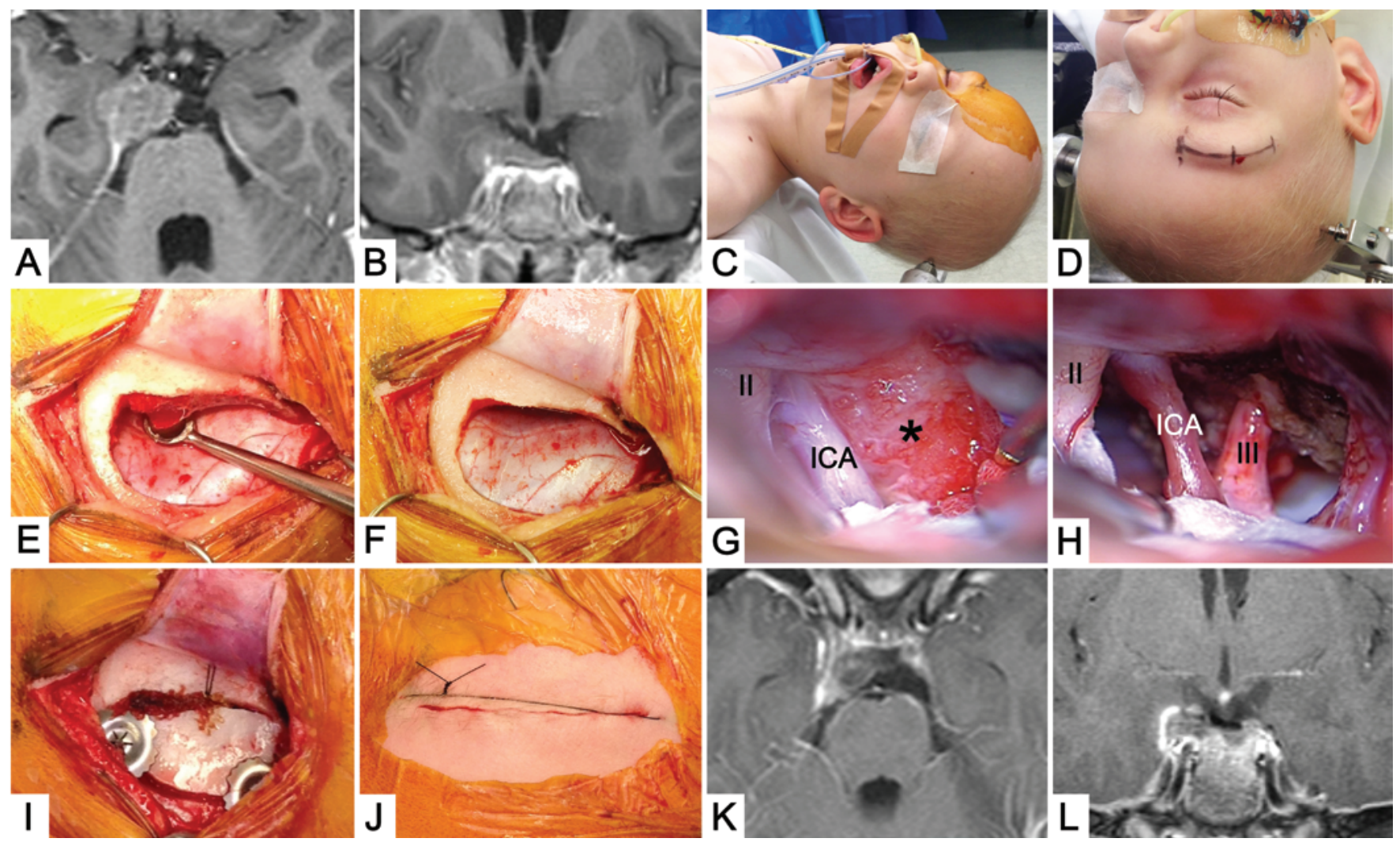

FIG. 1. Representative case showing use of supraorbital eyebrow approach in a 5 -year-old with a PNET of the parasellar area. A and B: Preoperative axial (A) and coronal (B) T1-weighted Gd-enhanced MR images revealing a right parasellar PNET. C and D: Proper positioning includes head extension (C) to allow the frontal lobe to fall away while the head is rotated toward the contralateral side (D). The skin incision resides in the upper aspect of the eyebrow and extends from the supraorbital notch (dashed line in D) to the lateral-most aspect of the eyebrow to expose the zygoma. The eyelid is closed with a temporary suture. $E$ and F: Supraorbital craniotomy $(E)$ and drilling of the orbital roof $(F)$ allow access to the subfrontal corridor. $\mathbf{G}$ and $H$ : The tumor (G, asterisk) is visualized around the ipsilateral optic nerve (II) and internal carotid artery (ICA) and resection (H) is performed around the optic and oculomotor nerves (III) as well as the ICA. I: The bone flap is replaced using low-profile titanium plates, ensuring that the bone flap is flush with the superior frontal bone edge. J: A subcuticular 4-0 nylon suture is used to approximate the skin edges. $\mathrm{K}$ and L: Postoperative axial $(\mathrm{K})$ and coronal $(\mathrm{L}) \mathrm{MR}$ images demonstrating subtotal resection of the right parasellar PNET. Figure is available in color online only.

Proper placement of this final skin suture is essential in obtaining a cosmetically acceptable result.

\section{Results}

\section{Clinical/Operative Outcomes}

A total of 54 children ( 35 boys and 19 girls) met the inclusion criteria (Table 1). The average age of patients at the time of surgery was 9.6 years (range $1.5-16$ years). The pathology consisted of craniopharyngiomas (39 cases), gliomas ( 6 cases), dermoids ( 2 cases), hypothalamic hamartomas ( 2 cases), a neurenteric cyst (1 case), trauma (1 case), a pituitary macroadenoma (1 case), cavernoma (1 case), and a primitive neuroectodermal tumor (PNET; 1 case). Lesions occurred in the following locations: suprasellar region (41 cases), optic pathway/hypothalamus (7 cases), cerebral peduncle ( 3 cases), parasellar region (1 case), orbit (1 case), and prepontine cistern (1 case). In all cases, surgery was performed with endoscopic assistance. In a total of 51 cases involving resectable lesions, 44 surgeries resulted in a gross-total resection and 7 cases resulted in subtotal resection. No recurrences have been identified to date. In a depressed skull fracture after trauma, a supraorbital eyebrow approach allowed elevation of the fracture, cranioplasty, and excellent cosmesis and skin closure. In no case was there a need to convert to a larger craniotomy or a new skin incision and different approach. Blood loss was minimal in all cases.

The approach provided access to the lesion in all cases without brain retraction (Fig. 3A-F). Brain retractors were not used in any case. The endoscope was used to assist and expand visualization or provide access to areas not reached by standard microscopic visualization in all cases (Fig. 2E and F). Within the small keyhole craniotomy, the lens shaft of the endoscope was stabilized by being placed against the bone edge. The endoscope was held by an assistant to allow for bimanual surgical technique or held by the senior surgeon for visual inspection (Fig. 2E and F). There were no endoscope-associated complications.

\section{Cosmetic Results}

In general, cosmesis was excellent (Table 2, Fig. 2I, Fig. 

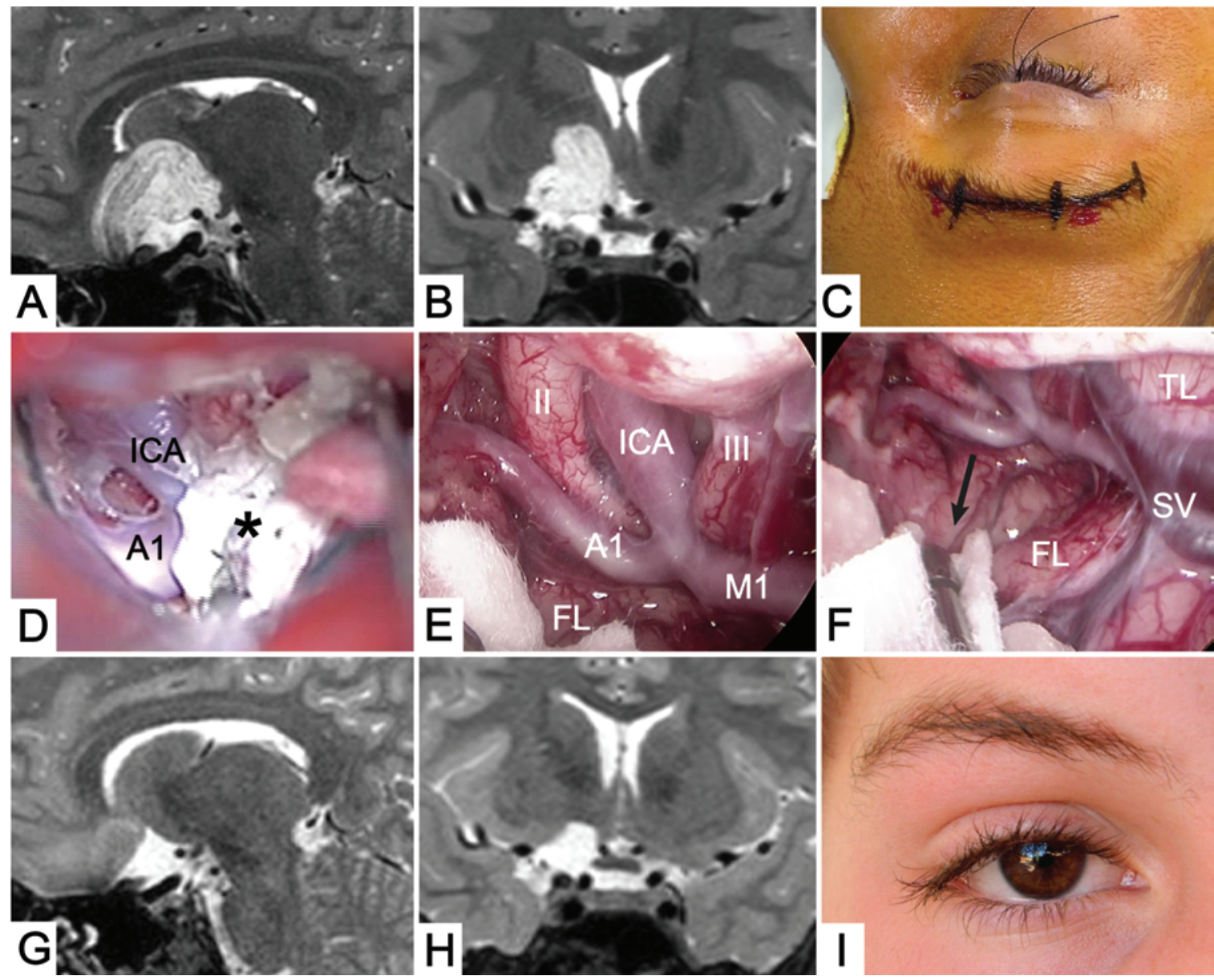

FIG. 2. Representative case showing use of endoscope to expand visualization and access to adjacent areas during the supraorbital eyebrow approach. A and B: Preoperative sagittal (A) and coronal (B) MR images revealing a right parasellar dermoid that extends superiorly into the basal frontal lobe. C: Skin incision along the superior aspect of the eyebrow. D: Microscopic resection of the dermoid (asterisk) around the ICA bifurcation and optic nerve (II). E and F: Endoscopic assisted resection and visualization of the optic nerve (II), ICA bifurcation, third nerve (III), sylvian fissure (SV), medial temporal lobe (TL), and basal frontal lobe (FL). Here the endoscopic views reveal the opened interchiasmatic cistern, optico-carotid cistern, carotid-oculomotor cistern and proximal sylvian fissure, which demonstrates the considerable working space provided by the approach without brain retraction. The endoscopic views also allow for optimal visualization, especially superiorly (black arrow), to confirm a complete resection. $\mathbf{G}$ and $\mathrm{H}$ : Postoperative sagittal $(\mathrm{G})$ and coronal $(\mathrm{H}) \mathrm{MRI}$ revealing resection of the dermoid. I: Photograph of the right eyebrow 6 months postoperatively with excellent cosmesis and imperceptible incision line or scar. Figure is available in color online only.

4A-G). Eyebrow alopecia and supraorbital hypoesthesia were not observed. Only 5 cases of frontalis palsy were identified. Forehead depression was not seen at 6-week follow-up in any case, but 2 years postoperatively 3 cases were identified; the depression was minor in appearance and less than $5 \mathrm{~mm}$ in depth from the skin surface in each of the 3 patients. In all cases, the incisional scar was barely visible 6 weeks postoperatively. In no case has a child complained about postoperative jaw or temple pain or pain with mastication.

\section{Complications}

There were few complications (Table 2). The frontal sinus is small in children, and no frontal sinus breach occurred. No CSF leak or wound infection was identified. Visual impairment occurred in 3 cases; 2 of the patients who experienced visual impairment had craniopharyn- giomas and the other had a cavernoma. Loss of smell was identified in only 1 child. Diabetes insipidus occurred in $85 \%$ of the patients with craniopharyngioma. Other complications included a complete third cranial nerve palsy after resection of a neurenteric cyst, panhypopituitarism after craniopharyngioma resection, and permanent hemiplegia after resection of a Grade III astrocytoma of the cerebral peduncle.

\section{Discussion}

\section{Evolution of the Supraorbital Eyebrow Approach}

Approaches to the anterior cranial fossa, medial temporal lobe, suprasellar and parasellar regions, and brainstem have evolved significantly over the last 3 decades with the goal of minimal brain retraction and optimal visualization. The supraorbital approach has been modified consid- 
TABLE 1. Demographic and clinical characteristics of 54 cases in which supraorbital eyebrow approaches were used in children

\begin{tabular}{lc}
\hline \multicolumn{1}{c}{ Characteristic } & Value* \\
\hline Age $(y r s)$ & 9.6 \\
\hline Mean & $1.5-16$ \\
\hline Range & $35(65)$ \\
\hline Male sex & \\
\hline Diagnosis & $39(72)$ \\
\hline Craniopharyngioma & $6(11)$ \\
\hline Glioma & $2(4)$ \\
\hline Dermoid & $2(4)$ \\
\hline Hypothalamic hamartoma & $1(2)$ \\
\hline Neurenteric cyst & $1(2)$ \\
\hline Trauma, compound skull fracture & $1(2)$ \\
\hline Pituitary macroadenoma, acromegaly & $1(2)$ \\
\hline Cavernoma & $1(2)$ \\
\hline PNET & \\
\hline Location & $41(76)$ \\
\hline Suprasellar & $7(13)$ \\
\hline Optic pathway/hypothalamus & $3(6)$ \\
\hline Cerebral peduncle & $1(2)$ \\
\hline Parasellar & $1(2)$ \\
\hline Orbit & $1(2)$ \\
\hline Prepontine cistern & $54(100)$ \\
\hline Surgery performed with endoscopic assistance & \\
\hline
\end{tabular}

* Values represent numbers of cases (\%) unless otherwise indicated.

erably to achieve this-initially by Jane et al. ${ }^{18}$ and subsequently by Delashaw et al. ${ }^{8}$ and Al Mefty and Fox. ${ }^{1}$ With advancements in neuroanesthesia and the advent of endoscope-assisted microneurosurgery, the keyhole concept developed, whereby only the necessary brain and surgical corridors that need exposure are exposed. ${ }^{13,25}$ Although the craniotomy is smaller, the access is the same., ${ }^{5,12}$ The minimization of the supraorbital craniotomy to expose only the anterolateral subfrontal working space has been effective. ${ }^{33}$ With proper tissue handling and surgical techniques, an eyebrow skin incision can be used to perform a supraorbital craniotomy, and thus tissue trauma can be limited and surgical time decreased. Concerns have been raised, however, regarding the use of this approach in pediatric cases. Here we reviewed all pediatric supraorbital eyebrow approaches performed by the senior author (C.T.) in cases that met inclusion criteria and found the approach highly effective in children in regard to multiple outcome measures.

\section{Working Space, Brain Retraction, and Use of the Endoscope}

Some have questioned using the supraorbital keyhole approach in children for various reasons, including insufficient working space. This concern is minimized by employing proper positioning and surgical technique; ${ }^{21}$ in all cases in our pediatric series, there was an unimpeded view and access to the pathology of interest. Additionally, others have expressed concern over excessive brain retraction
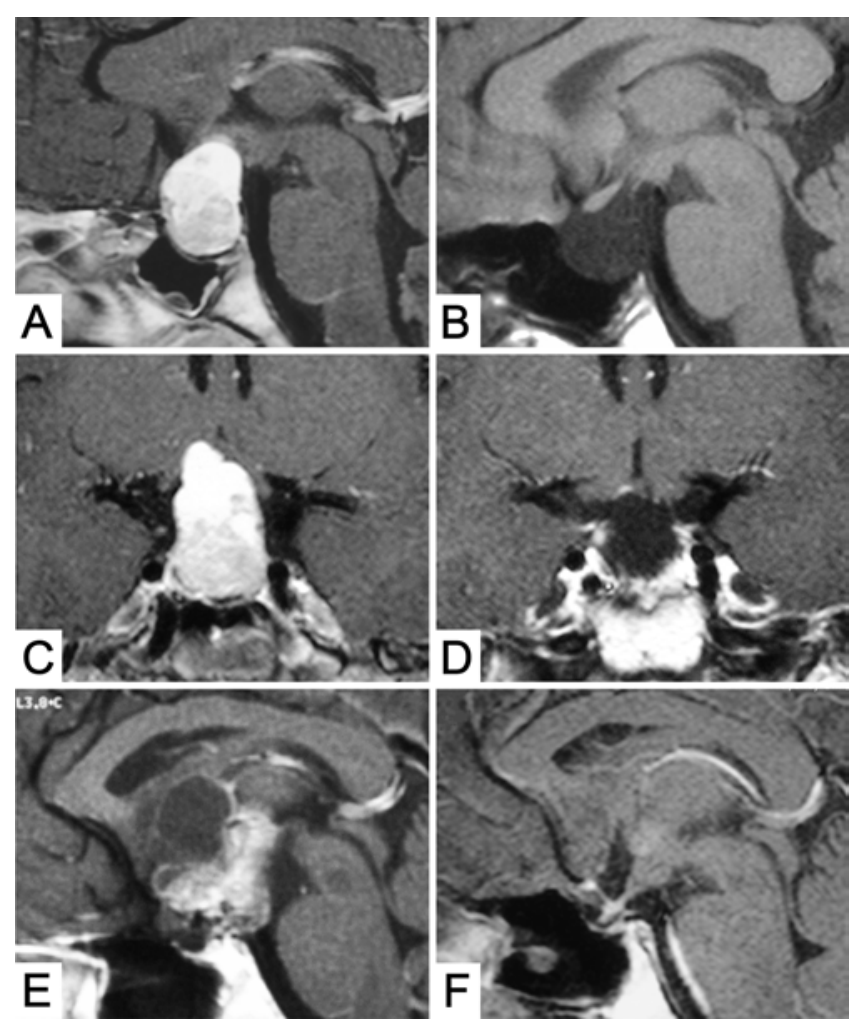

FIG. 3. Representative pre- and postoperative T1-weighted Gdenhanced MR images obtained in patients who underwent resection of large craniopharyngiomas via a supraorbital eyebrow approach. Gross-total resection was achieved in all 3 of the cases shown here, demonstrating that this approach can provide adequate working space and visualization for complete resection of these lesions. A and B: Preoperative $(A)$ and postoperative $(B)$ sagittal MR images obtained in a 10-year-old boy. C and D: Preoperative (C) and postoperative (D) coronal MR images obtained in a 12-year-old girl. $E$ and $F$ : Preoperative $(E)$ and postoperative $(F)$ sagittal MR images obtained in an 8-year-old boy.

with the supraorbital keyhole approach. ${ }^{34}$ However, just as with the classic approach, proper positioning allows the frontal lobe to fall away so that the supraorbital keyhole approach gives direct anterolateral subfrontal access to the interchiasmatic cistern, optico-carotid cistern, carotidoculomotor cistern, and proximal sylvian fissure. ${ }^{8,18}$ Opening of these cisterns and CSF spaces with an arachnoid knife permits CSF egress and allows the frontal lobe to fall completely away from view. In our series, excess brain retraction was not observed in any of the 54 cases, and there was no need to use brain retractors in any case.

If one is concerned about brain retraction, the orbital roof can be removed to provide working space typically occupied by the orbit, 2,5,13,14,16,20,24,28 but this is often not necessary. Removal of the orbital roof provides greater upward angulation, which can be helpful in obtaining access to anterior communicating aneurysms or suprasellar lesions extending superiorly into the third ventricle. Additionally, a $30^{\circ}$ endoscope can be used to visualize superiorly, ${ }^{32}$ and using angled instruments, the lesion can be treated with standard bimanual surgical technique (Fig. $2 \mathrm{E}$ and $\mathrm{F}$ ). When this endoscopic technique is utilized, the orbital rim may not need removal. In general, the endoscope is an essential adjunct that extends the visualization 
TABLE 2. Clinical outcomes and complications for 54 supraorbital eyebrow approaches in children

\begin{tabular}{|c|c|}
\hline Characteristic & Value* $^{*}$ \\
\hline \multicolumn{2}{|l|}{ Follow-up (yrs) } \\
\hline Mean & 8.9 \\
\hline Range & $0.5-16$ \\
\hline \multicolumn{2}{|l|}{ Extent of tumor resection $(n=51)$} \\
\hline Gross-total & $44(86)$ \\
\hline Subtotal & $7(14)$ \\
\hline Partial & $0(0)$ \\
\hline \multicolumn{2}{|l|}{ Cosmesis $(n=42)$} \\
\hline Eyebrow alopecia & $0(0)$ \\
\hline Frontalis palsy & $5(12)$ \\
\hline Supraorbital hypesthesia & $0(0)$ \\
\hline \multicolumn{2}{|l|}{ Forehead depression } \\
\hline 6-wk follow-up & $0(0)$ \\
\hline 6-mo follow-up & $1(2)$ \\
\hline 2-yr follow-up & $3(7)$ \\
\hline Titanium plate migration & $0(0)$ \\
\hline \multicolumn{2}{|l|}{ Complications $(n=54)$} \\
\hline Frontal sinus breach & $0(0)$ \\
\hline CSF leak & $0(0)$ \\
\hline Wound infection & $0(0)$ \\
\hline Visual impairment & $3(6)$ \\
\hline Anosmia & $1(2)$ \\
\hline \multicolumn{2}{|l|}{ Diabetes insipidus } \\
\hline Craniopharyngioma $(n=39)$ & $33(85)$ \\
\hline Hypothalamic hamartoma $(n=2)$ & $2(100) \dagger$ \\
\hline Pituitary adenoma $(n=1)$ & $1(100) \dagger$ \\
\hline Other & $3(6)$ \\
\hline
\end{tabular}

* Values represent numbers of cases (\%) unless otherwise indicated.

$\dagger$ These 3 cases resulted in transient diabetes insipidus. of the supraorbital approach beyond the view of the operating microscope.

\section{Appropriately Selected Cases and Effectiveness of the Approach}

It is important to consider each case on an individual basis, as the imaging characteristics of each pathology and individual anatomical variation strongly influence whether a lesion is treatable via a particular approach. Even then, the optimal approach is debatable and may depend on the surgeon's familiarity with the possible approaches. However, the surgeon must understand the anatomical limitations of each approach. ${ }^{10,21,35}$ As seen with our pediatric case series, gross-total resection was achieved in $84 \%$ of resectable lesions, of which a large majority were craniopharyngiomas, and no recurrences have been noted to date. Although these results are better than published series, they do correspond with results of craniopharyngioma management previously reported by the senior author. ${ }^{32} \mathrm{~A}$ possible explanation is the benefit of endoscope-assisted surgery in detecting "hidden" remnants and consequently allowing for more comprehensive and complete resections. In no case did the supraorbital eyebrow approach limit the ability to achieve gross-total resection, and this is a testament to using the approach in properly selected cases. ${ }^{32,35}$ For lesions that extend considerably from the parasellar region into the temporal fossa, we use a traditional pterional ${ }^{36}$ or mini-pterional $l^{11}$ approach, as access to the temporal fossa is limited in the supraorbital approach. In cases in which the lesion is suprasellar in location but retrochiasmal, as can occur with craniopharyngioma, an extended endonasal transsphenoidal approach would be considered, and its advantages and disadvantages would be compared with those of the supraorbital eyebrow approach. ${ }^{10}$

\section{Cosmesis}

Some have expressed concern over the cosmetic outcome achieved with an eyebrow skin incision, especially in children. ${ }^{30,34}$ Placing the incision at the superior aspect of the eyebrow hides the incision within the brow line, and with proper tissue handling and closure, the incision line

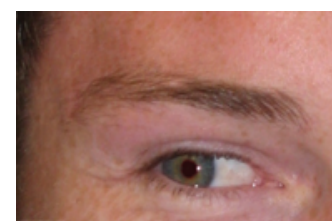

A

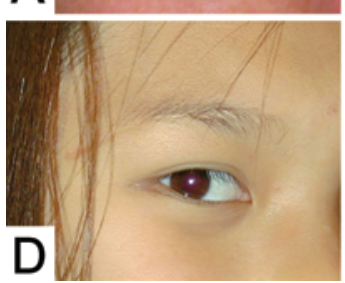

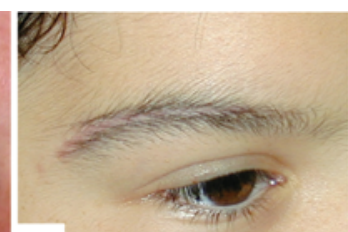

B

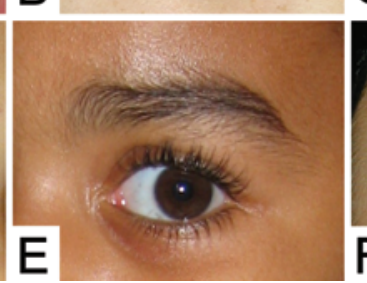

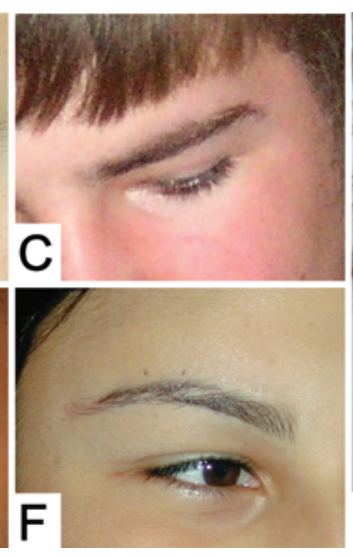

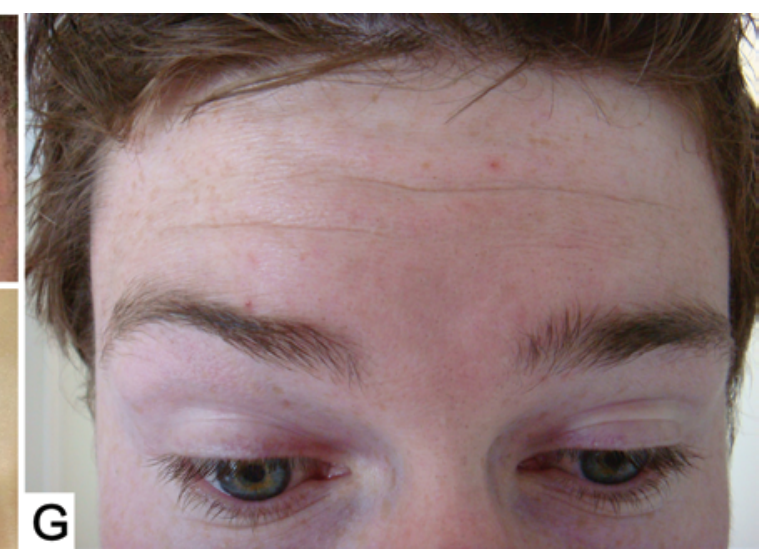

FIG. 4. Postoperative eyebrow photographs demonstrating typical cosmetic outcome. A-F: Photographs of eyebrow skin incisions in children of various ethnicities at least 6 weeks after surgery demonstrating no loss of hair and an undetectable scar. G: Can you tell which side the approach was performed on in this patient? This photograph was taken after a right supraorbital eyebrow approach. The patient has an undetectable scar and normal frontalis function. Figure is available in color online only. 
and scar are practically undetectable 6 weeks postoperatively. This was observed with a variety of skin types, given the ethnic diversity of the children in our series. Others, who have had patients with thin light-colored eyebrows, express concern over the eyebrow incision..$^{15}$ Even in cases involving children with very thin eyebrows, we have not had complaints from patients or their families. Others have used a skin incision above the eyebrow to avoid the hair loss potentially associated with an incision in the eyebrow itself. We do not feel this is as cosmetically pleasing and use an incision within the superior aspect of the brow. As seen in our results, we have not observed loss of hair with this incision (Table 2, Fig. 4). In the growing pediatric calvaria, any interference with the dynamic nature of the sutures might cause long-term growth problems and subsequent cosmetic deformity. However, no bone defect over the pterion, abnormal bone growth, or migration of the titanium plates was observed. However, a minor bone defect on the forehead where the craniotomy cut was made was observed in 3 cases. Because the supraorbital approach utilizes a small incision and involves minimal temporalis dissection, scalp pain, temporalis atrophy, and difficulty with mastication were rarely observed. Important in adults, this may be even more of an advantage in the very young.

\section{Complications}

Other concerns have included risk of frontal sinus violation, CSF leak, and risk of infection and meningitis. ${ }^{26}$ Even in our adult series, we avoid the frontal sinus, and infection is almost nonexistent. In children, the frontal sinus is generally small, and we did not encounter it in any case in our series (Table 2).

\section{Limitations}

As with all retrospective studies, there is an inherent bias compared with prospective studies. There was incomplete follow-up of cosmetic outcome, as only $78 \%$ of the patients had direct observation and recorded findings.

\section{Conclusions}

We demonstrated in the largest series to date that the supraorbital eyebrow approach could be successfully used in properly selected cases in patients of all pediatric ages with excellent cosmesis and minimal complications.

\section{References}

1. Al-Mefty O, Fox JL: Superolateral orbital exposure and reconstruction. Surg Neurol 23:609-613, 1985

2. Bognár L, Czirják S, Madarassy G: Frontolateral keyhole craniotomy through a superciliary skin incision in children. Childs Nerv Syst 19:765-768, 2003

3. Cavalcanti DD, García-González U, Agrawal A, Crawford NR, Tavares PL, Spetzler RF, et al: Quantitative anatomic study of the transciliary supraorbital approach: benefits of additional orbital osteotomy? Neurosurgery 66 (6 Suppl Operative):205-210, 2010

4. Chalouhi N, Jabbour P, Ibrahim I, Starke RM, Younes P, El Hage G, et al: Surgical treatment of ruptured anterior circulation aneurysms: comparison of pterional and supraorbital keyhole approaches. Neurosurgery 72:437-442, 2013
5. Cheng CM, Noguchi A, Dogan A, Anderson GJ, Hsu FP, McMenomey SO, et al: Quantitative verification of the keyhole concept: a comparison of area of exposure in the parasellar region via supraorbital keyhole, frontotemporal pterional, and supraorbital approaches. Laboratory investigation. J Neurosurg 118:264-269, 2013

6. Czirják S, Szeifert GT: Surgical experience with frontolateral keyhole craniotomy through a superciliary skin incision.

Neurosurgery 48:145-150, 2001

7. Dare AO, Landi MK, Lopes DK, Grand W: Eyebrow incision for combined orbital osteotomy and supraorbital minicraniotomy: application to aneurysms of the anterior circulation. Technical note. J Neurosurg 95:714-718, 2001

8. Delashaw JB Jr, Jane JA, Kassell NF, Luce C: Supraorbital craniotomy by fracture of the anterior orbital roof. Technical note. J Neurosurg 79:615-618, 1993

9. El Shafei HI: Anterolateral mini fronto-orbito-zygomatic craniotomy via an eyebrow incision in pediatrics: technical notes and evaluation. Pediatr Neurosurg 47:248-253, 2011

10. Fatemi N, Dusick JR, de Paiva Neto MA, Malkasian D, Kelly DF: Endonasal versus supraorbital keyhole removal of craniopharyngiomas and tuberculum sellae meningiomas. Neurosurgery 64 (5 Suppl 2):269-286, 2009

11. Figueiredo EG, Deshmukh P, Nakaji P, Crusius MU, Crawford N, Spetzler RF, et al: The minipterional craniotomy: technical description and anatomic assessment. Neurosurgery 61 (5 Suppl 2):256-265, 2007

12. Figueiredo EG, Deshmukh V, Nakaji P, Deshmukh P, Crusius MU, Crawford N, et al: An anatomical evaluation of the mini-supraorbital approach and comparison with standard craniotomies. Neurosurgery 59 (4 Suppl 2):ONS212ONS220, 2006

13. Fries G, Perneczky A: Endoscope-assisted brain surgery: part 2 -analysis of 380 procedures. Neurosurgery 42:226-232, 1998

14. Hayhurst C, Teo C: Tuberculum sella meningioma. Otolaryngol Clin North Am 44:953-963, viii-ix, 2011

15. Hernesniemi J, Ishii K, Niemelä M, Smrcka M, Kivipelto L, Fujiki M, et al: Lateral supraorbital approach as an alternative to the classical pterional approach. Acta Neurochir Suppl 94:17-21, 2005

16. Jallo GI, Bognar L: Eyebrow surgery: the supraciliary craniotomy: technical note. Neurosurgery 59 (1 Suppl 1): ONSE157-ONSE158, 2006

17. Jallo GI, Suk I, Bognár L: A superciliary approach for anterior cranial fossa lesions in children. Technical note. J Neurosurg 103 (1 Suppl):88-93, 2005

18. Jane JA, Park TS, Pobereskin LH, Winn HR, Butler AB: The supraorbital approach: technical note. Neurosurgery 11:537-542, 1982

19. Jho HD: Orbital roof craniotomy via an eyebrow incision: a simplified anterior skull base approach. Minim Invasive Neurosurg 40:91-97, 1997

20. Kanaan IN: Trans-eyebrow mini-orbitozygomatic pterional approach for minimally invasive skull base surgery. Minim Invasive Neurosurg 48:34-38, 2005

21. Little ASGP, Darbar A, Teo C: Supraorbital eyebrow approach: a less invasive corridor to lesions of the anterior cranial fossa, parasellar region, and ventral brainstem, in Cappabianca P, Califano L, Iaconetta G (eds): Cranial, Craniofacial and Skull Base Surgery. Milan: Springer-Verlag, 2010, pp 27-38

22. Maus M, Goldman HW: Removal of orbital apex hemangioma using new transorbital craniotomy through suprabrow approach. Ophthal Plast Reconstr Surg 15:166-170, 1999

23. Park J, Kang DH, Chun BY: Superciliary keyhole surgery for unruptured posterior communicating artery aneurysms with oculomotor nerve palsy: maximizing symptomatic resolution and minimizing surgical invasiveness. Clinical article. J Neurosurg 115:700-706, 2011 
24. Park J, Woo H, Kang DH, Sung JK, Kim Y: Superciliary keyhole approach for small unruptured aneurysms in anterior cerebral circulation. Neurosurgery 68 (2 Suppl Operative):300-309, 2011

25. Perneczky A, Fries G: Endoscope-assisted brain surgery: part 1 - evolution, basic concept, and current technique. Neurosurgery 42:219-225, 1998

26. Ramos-Zúñiga R, Velázquez H, Barajas MA, López R, Sánchez E, Trejo S: Trans-supraorbital approach to supratentorial aneurysms. Neurosurgery 51:125-131, 2002

27. Reisch R, Perneczky A: Ten-year experience with the supraorbital subfrontal approach through an eyebrow skin incision. Neurosurgery 57 (4 Suppl):242-255, 2005

28. Reisch R, Perneczky A, Filippi R: Surgical technique of the supraorbital key-hole craniotomy. Surg Neurol 59:223-227, 2003

29. Reisch R, Stadie A, Kockro R, Gawish I, Schwandt E, Hopf $\mathrm{N}$ : The minimally invasive supraorbital subfrontal key-hole approach for surgical treatment of temporomesial lesions of the dominant hemisphere. Minim Invasive Neurosurg 52:163-169, 2009

30. Shanno G, Maus M, Bilyk J, Schwartz S, Savino P, Simeone $\mathrm{F}$, et al: Image-guided transorbital roof craniotomy via a suprabrow approach: a surgical series of 72 patients. Neurosurgery 48:559-568, 2001

31. Steiger HJ, Schmid-Elsaesser R, Stummer W, Uhl E: Transorbital keyhole approach to anterior communicating artery aneurysms. Neurosurgery 48:347-352, 2001

32. Teo C: Application of endoscopy to the surgical management of craniopharyngiomas. Childs Nerv Syst 21:696-700, 2005
33. van Lindert E, Perneczky A, Fries G, Pierangeli E: The supraorbital keyhole approach to supratentorial aneurysms: concept and technique. Surg Neurol 49:481-490, 1998

34. Warren WL, Grant GA: Transciliary orbitofrontozygomatic approach to lesions of the anterior cranial fossa. Neurosurgery 64 (5 Suppl 2):324-330, 2009

35. Wilson DA, Duong H, Teo C, Kelly DF: The supraorbital endoscopic approach for tumors. World Neurosurg 82:e243e256, 2014

36. Yasargil MG, Antic J, Laciga R, Jain KK, Hodosh RM, Smith RD: Microsurgical pterional approach to aneurysms of the basilar bifurcation. Surg Neurol 6:83-91, 1976

\section{Author Contributions}

Conception and design: all authors. Acquisition of data: Dlouhy, Teo. Analysis and interpretation of data: Dlouhy, Teo. Drafting the article: Dlouhy. Critically revising the article: all authors. Reviewed submitted version of manuscript: all authors. Approved the final version of the manuscript on behalf of all authors: Dlouhy. Administrative/technical/material support: Dlouhy, Teo. Study supervision: Teo.

\section{Correspondence}

Brian J. Dlouhy, Department of Neurosurgery, University of Iowa Hospitals and Clinics, 200 Hawkins Dr., Iowa City, IA 52242. email: brian-dlouhy@uiowa.edu. 